\title{
COMPLEMENTARY TRIANGULAR FORMS
}

\author{
ROB ZUIDWIJK \\ Center for Mathematics and Informatics \\ P.O. Box 94079, NL-1090 GB, The Netherlands \\ E-mail: zuidwijk@cwi.nl
}

\begin{abstract}
The notion of simultaneous reduction of pairs of matrices and linear operators to triangular forms is introduced and a survey of known material on the subject is given. Further, some open problems are pointed out throughout the text. The paper is meant to be accessible to the non-specialist and does not contain any new results or proofs.
\end{abstract}

1. Background information. The well-known theorem of Schur (see for example [23]) states that if $A$ is a complex $m \times m$ matrix, then there exists a unitary $m \times m$ matrix $U$, such that $U^{-1} A U$ is an upper triangular matrix. In other words, each square complex matrix can be reduced to upper triangular form by a unitary similarity transformation. For pairs of square complex matrices, the following result was obtained by McCoy [24].

Theorem 1. Let $A, Z$ be a pair of complex $m \times m$ matrices. Then the following two statements are equivalent:

1. There exists an invertible $m \times m$ matrix $S$, such that both $S^{-1} A S$ and $S^{-1} Z S$ are upper triangular matrices.

2. For each polynomial $p(\lambda, \mu)$ in the non-commuting variables $\lambda$ and $\mu$, the $m \times m$ matrix $p(A, Z)(A Z-Z A)$ is nilpotent.

A pair of $m \times m$ matrices $A, Z$ which satisfies the statements of Theorem 1 is said to admit simultaneous reduction to upper triangular form. The proof of Theorem 1 in [24] is rather involved. Elementary proofs of this theorem have been obtained in [13] and [17]. The theorem is made more explicit for certain pairs of matrices in [19] and [20]. Further, a recent extension of Theorem 1 is given in [26]. The literature on this subject, which includes a paper of Frobenius [16] of almost a century ago, is extensive. For more information and references, see [21]. Generalizations of Theorem 1 to an infinite dimensional context has been obtained in [22] and [25]; see also Section 3.

1991 Mathematics Subject Classification: Primary 15A21; Secondary 47A68.

The paper is in final form and no version of it will be published elsewhere. 
This paper also deals with simultaneous reduction to triangular forms. However, the emphasis is not on simultaneous reduction to "the same", e.g. upper, triangular form, but on simultaneous reduction to "complementary" triangular forms. As we shall see later on, the motivation for studying this issue comes from systems theory. But let us first give the definition and state two of the earliest results.

Let $A$ and $Z$ be $m \times m$ matrices. We say that the pair $A, Z$ admits simultaneous reduction to complementary triangular forms, if there exists an invertible matrix $S$ (not necessarily unitary) such that $S^{-1} A S$ is an upper triangular matrix and $S^{-1} Z S$ is a lower triangular matrix.

Here are two early results about this notion. The first result was implicit in the proof of Theorem 1.6 in [3]; for an explicit statement, see [2]. The second result appeared in [4]. The spectrum of a square matrix $A$ is denoted by $\sigma(A)$.

Theorem 2. Let $A, Z$ be a pair of $m \times m$ matrices. If either $A$ or $Z$ is diagonalizable, then the pair $A, Z$ admits simultaneous reduction to complementary triangular forms.

Theorem 3. Let $A, Z$ be a pair of $m \times m$ matrices, such that $\operatorname{rank}(A-Z)=1$ and such that $\sigma(A) \cap \sigma(Z)=\emptyset$. Then the pair $A, Z$ admits simultaneous reduction to complementary triangular forms.

If a pair of $m \times m$ matrices $A, Z$ admits simultaneous reduction to upper triangular form, we may assume without loss of generality that the invertible $m \times m$ matrix $S$ involved can be chosen to be unitary. For complementary triangular forms, this is not the case: There exist pairs of $m \times m$ matrices $A, Z$ that admit simultaneous reduction to complementary triangular forms, for which the invertible $m \times m$ matrix $S$ involved cannot be taken unitary. This already indicates that simultaneous reduction to upper triangular form and simultaneous reduction to complementary triangular forms are quite different matters. Nevertheless, a connection between the two notions is formulated in the following proposition, which is proved in [12].

Proposition 4. Let $A$ and $Z$ be $m \times m$ matrices. The pair $A, Z$ admits simultaneous reduction to complementary triangular forms if and only if there exists a positive definite $m \times m$ matrix $H$ such that the pair $A, H^{-1} Z^{*} H$ admits simultaneous reduction to upper triangular form.

The collection of pairs of $m \times m$ matrices that admit simultaneous reduction to complementary triangular forms is denoted by $\mathcal{C}(m)$. The collections of low order matrices $\mathcal{C}(2)$ and $\mathcal{C}(3)$ have been described completely; see Remark 2.3.6 in [29]. For $m \geq 4$, a full description of $\mathcal{C}(m)$ is not known. One could hope for a reasonable general description by combining Proposition 4 and McCoy's theorem (Theorem 1). Unfortunately, the existence problem of the positive definite matrix $H$ in Proposition 4 turns out to be as complicated as the study of simultaneous reduction to complementary triangular forms itself.

Altogether, there are no non-trivial results concerning the general case. On the other hand, some quite satisfactory results are obtained for pairs of matrices, taken from certain classes of matrices. Results in this direction have been obtained by several authors; see [4], [8]-[11] and [15]. One of these results concerns pairs of matrices of first companion 
type, i.e., matrices of the form

$$
C_{a}=\left(\begin{array}{ccccc}
0 & 1 & & & \\
& 0 & \ddots & & \\
& & \ddots & 1 & \\
-a_{0} & -a_{1} & \cdots & -a_{m-2} & -a_{m-1}
\end{array}\right) .
$$

Note that the characteristic polynomial for this matrix reads $a(\lambda)=\operatorname{det}\left(\lambda-C_{a}\right)=$ $a_{0}+a_{1} \lambda+\ldots+a_{m-1} \lambda^{m-1}+\lambda^{m}$. The following result is taken from [4].

Theorem 5. Let $A$ and $Z$ be first companion $m \times m$ matrices. Then the pair $A, Z$ admits simultaneous reduction to complementary triangular forms if and only if there exist orderings

$$
\alpha_{1}, \ldots, \alpha_{m}, \quad \zeta_{1}, \ldots, \zeta_{m}
$$

of the eigenvalues for $A$ and $Z$ respectively, counted according to their algebraic multiplicities, such that

$$
\alpha_{k} \neq \zeta_{l}, \quad k+l \leq m
$$

In short, a pair of first companion matrices admits simultaneous reduction to complementary triangular forms if and only if a combinatorial condition is met. Moreover, this combinatorial condition comes up in the study of the Two Machine Flow Shop Problem from job scheduling theory. For more details on this surprising connection, see [5]-[7], [31].

Now let us give a motivation for studying complementary triangular forms for pairs of matrices. We shall do this by making a connection with systems theory. Consider the linear dynamical system

$$
(\Sigma)\left\{\begin{array}{l}
x^{\prime}(t)=A x(t)+B u(t) \\
y(t)=C x(t)+u(t) \\
x(0)=0
\end{array} \quad t \geq 0\right.
$$

where $x(t)$ is an $m$-vector, $u(t)$ and $y(t)$ are $n$-vectors, and $A, B$ and $C$ are matrices of appropriate sizes. The equations represent a system $\Sigma$ with input $u(t)$ and output $y(t)$ (at time $t$ ) as illustrated below.

$$
u \longrightarrow \Sigma \longrightarrow y
$$

By taking the Laplace transform of (1), and by cancelling the Laplace transform $\widehat{x}(\lambda)$ of $x(t)$, we see that the Laplace transforms $\widehat{u}(\lambda)$ and $\widehat{y}(\lambda)$ of, respectively, the input vector $u(t)$ and the output vector $y(t)$ are related as follows:

$$
\widehat{y}(\lambda)=W(\lambda) \widehat{u}(\lambda) .
$$

Here $W(\lambda)$ is the so-called transfer function of $\Sigma$, given by

$$
W(\lambda)=I_{n}+C\left(\lambda I_{m}-A\right)^{-1} B .
$$

The $n \times n$ matrix function $W(\lambda)$ is rational, i.e., its entries are quotients of polynomials. Further, $W(\lambda)$ is analytic at infinity with value $W(\infty)=I_{n}$, the $n \times n$ identity matrix. In the following, we shall consider factorizations of such transfer functions which lead to cascarle decompositions of the underlying systems. 
It can be shown that all rational $n \times n$ matrix functions $W(\lambda)$ analytic at infinity with $W(\infty)=I_{n}$ can be written in realization form (2) for certain matrices $A, B$ and $C$. The smallest possible integer $m$ for which a given rational matrix function $W(\lambda)$ admits a realization (2) is called the McMillan degree of $W(\lambda)$ and is denoted by $\delta(W)$. One may interpret the integer $\delta(W)$ as a measure of complexity of the corresponding system $\Sigma$. In fact, it equals the number of poles of $W(\lambda)$ counted according to their pole multiplicities (cf. [3]).

To put factorizations of these rational matrix functions in perspective, we will first discuss the scalar case: The quotient $w(\lambda)=p(\lambda) / q(\lambda)$ of two polynomials of degree $m$ is a scalar rational function. We will assume that the polynomials are monic, i.e., have leading coefficient equal to one, so that $w(\infty)=1$. The scalar function $w(\lambda)$ has McMillan degree $\delta(w)=m$ if and only if $p(\lambda)$ and $q(\lambda)$ have no roots in common. The Fundamental Theorem of Algebra, applied both to $p(\lambda)$ and $q(\lambda)$, then yields

$$
w(\lambda)=\left(\frac{\lambda-\alpha_{1}}{\lambda-\beta_{1}}\right)\left(\frac{\lambda-\alpha_{2}}{\lambda-\beta_{2}}\right) \ldots\left(\frac{\lambda-\alpha_{m}}{\lambda-\beta_{m}}\right),
$$

where $\alpha_{1}, \ldots, \alpha_{m}$ are the roots of $p(\lambda)$, and $\beta_{1}, \ldots, \beta_{m}$ are the roots of $q(\lambda)$. Note that

$$
w_{k}(\lambda)=\frac{\lambda-\alpha_{k}}{\lambda-\beta_{k}}=1+\frac{\beta_{k}-\alpha_{k}}{\lambda-\beta_{k}}, \quad k=1, \ldots, m,
$$

are scalar rational functions of McMillan degree one. Therefore, each scalar rational function $w(\lambda)$ of McMillan degree $m$, with $w(\infty)=1$, is the product

$$
w(\lambda)=w_{1}(\lambda) \ldots w_{m}(\lambda)
$$

of $m$ scalar rational functions of McMillan degree one.

We will now discuss this type of factorization for rational matrix functions. Rational matrix functions of McMillan degree one are called elementary rational matrix functions. Such a function is of the form

$$
W(\lambda)=I_{n}+\frac{1}{\lambda-\alpha} c b^{T},
$$

where $\alpha$ is a scalar and $b$ and $c$ are $n$-vectors. A complete factorization of a minimal realization $(2)$ is a factorization

$$
W(\lambda)=W_{1}(\lambda) \ldots W_{m}(\lambda)
$$

into $m=\delta(W)$ elementary rational matrix functions

$$
W_{k}(\lambda)=I_{n}+\frac{1}{\lambda-\alpha_{k}} c_{k} b_{k}^{T}, \quad k=1, \ldots, m
$$

Each elementary rational matrix function $W_{k}(\lambda)$ corresponds to an "elementary" system $\Sigma_{k}$. In this manner, the complete factorization (4) corresponds to the cascade decomposition

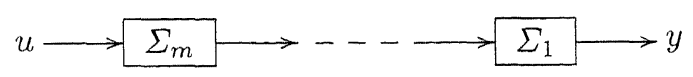

of the system $\Sigma$ into "elementary" systems $\Sigma_{1}, \ldots, \Sigma_{m}$. The question of which rational matrix functions admit a complete factorization is answered by the following theorem, which appeared in [2] and [4].

THEOREM 6. A rational matrix function $W(\lambda)=I_{n}+C\left(\lambda I_{m}-A\right)^{-1} B$ of McMillan degree $m$ admits a complete factorization if and only if the pair of $m \times m$ matrices $A$, $A-B C$ admits simultaneous reduction to complementary triangular forms. 
Not all rational matrix functions admit a complete factorization. For example, the rational $2 \times 2$ matrix function

$$
W(\lambda)=\left(\begin{array}{cc}
1 & -1 / \lambda^{2} \\
0 & 1
\end{array}\right)
$$

does not have this property. Indeed, this rational matrix function admits a minimal realization (2) with

$$
A=\left(\begin{array}{ll}
0 & 1 \\
0 & 0
\end{array}\right), \quad B=\left(\begin{array}{ll}
0 & 0 \\
0 & 1
\end{array}\right), \quad C=\left(\begin{array}{cc}
-1 & 0 \\
0 & 0
\end{array}\right) .
$$

Computation yields $A=A-B C$. For the pair $A, A-B C$ to admit simultaneous reduction to complementary triangular forms, it is necessary and sufficient that $A=A-B C$ is diagonalizable. Clearly, this is not the case. Theorem 6 implies that $W(\lambda)$ does not admit a complete factorization.

So far, known material concerning complementary triangular forms and its connection with systems theory has been presented. In the next two sections, an outline is given of more recent results on the subject. These results and their proofs can be found in [29].

2. Finite matrices. The first result we shall discuss here is closely related to Theorem 6 . As mentioned in the previous section, not all rational matrix functions admit a complete factorization. Recall that in the definition of a complete factorization, we required the elementary factors to be of a specific type, as in (3). If more general types of elementary factors are allowed (e.g. non-square ones), then the situation becomes different; see [28].

The factorization result we are about to present also involves elementary factors of the specific form (3), as in the case of complete factorization. However, we will allow the number of elementary factors to be larger than the McMillan degree of the rational matrix function under consideration. In terms of [3], this means that the factorizations need not be minimal. The smallest number of factors that is needed to factorize a given rational matrix function $W(\lambda)$ into elementary factors is denoted by $\varrho(W)$, and a factorization into $\varrho(W)$ factors is called a quasicomplete factorization. We now state the factorization result.

THEOREM 7. Each non-trivial rational matrix function

$$
W(\lambda)=I_{n}+C\left(\lambda I_{m}-A\right)^{-1} B
$$

admits a quasicomplete factorization. In fact, the number of factors involved in such a factorization satisfies the estimates

$$
\delta(W) \leq \varrho(W) \leq 2 \delta(W)-1 .
$$

The first inequality in (5) is obvious, the second one requires a non-trivial proof (see [29] or [30]) that uses the Pole Assignment Theorem from systems theory. Another aspect of the proof is that it starts with a minimal realization $W(\lambda)=I_{n}+C\left(\lambda I_{m}-A\right)^{-1} B$, from which another (non-minimal) realization $W(\lambda)=I_{n}+\widetilde{C}\left(\lambda I_{\varrho}-\widetilde{A}\right)^{-1} \widetilde{B}$ is constructed. The matrices $\widetilde{A}, \widetilde{B}$ and $\widetilde{C}$ are particular extensions of the matrices $A, B$ and $C$, constructed in such a way that the pair $\widetilde{A}, \widetilde{A}-\widetilde{B} \widetilde{C}$ admits simultaneous reduction to complementary triangular forms. 
A special type of extensions of those mentioned in the last paragraph are extensions with zeroes. This type of extensions leads to the following problem, which, by the way, also comes up in the study of complementary triangular forms in an infinite-dimensional context; see Section 3.

Let $A_{1}$ and $Z_{1}$ be $m_{1} \times m_{1}$ matrices. Does there exist a non-negative integer $m_{2}$, such that the pair of $\left(m_{1}+m_{2}\right) \times\left(m_{1}+m_{2}\right)$ matrices $A_{1} \oplus O_{m_{2}}, Z_{1} \oplus O_{m_{2}}$ admits simultaneous reduction to complementary triangular forms? In other words, does the pair $A_{1}, Z_{1}$ admit simultaneous reduction to complementary triangular forms after extension with zeroes?

Note that we include the case when the pair $A_{1}, Z_{1}$ admits simultaneous reduction to complementary triangular forms without extension, i.e., the case when the integer $m_{2}$ can be taken zero.

It is a highly non-trivial fact that there exist pairs of $m_{1} \times m_{1}$ matrices $A_{1}, Z_{1}$ which do not admit simultaneous reduction to complementary triangular forms, but obtain this property after extension with zeroes. Indeed, the pair of $4 \times 4$ matrices

$$
A_{1}=\left(\begin{array}{llll}
0 & 1 & 0 & 0 \\
0 & 0 & 0 & 0 \\
1 & 0 & 0 & 0 \\
0 & 1 & 0 & 0
\end{array}\right), \quad Z=\left(\begin{array}{llll}
0 & 1 & 0 & 0 \\
0 & 0 & 1 & 0 \\
0 & 0 & 0 & 1 \\
0 & 0 & 0 & 0
\end{array}\right)
$$

does not admit simultaneous reduction to complementary triangular forms, i.e., $\left(A_{1}, Z_{1}\right)$ $\notin \mathcal{C}(4)$. On the other hand, $\left(A_{1} \oplus 0, Z_{1} \oplus 0\right) \in \mathcal{C}(5)$. Details are given in Section 3.2 of [29].

In Chapter 3 of [29], it is shown that for all pairs of matrices that are taken from classes of matrices for which a transparent description of simultaneous reduction to complementary triangular forms is known, e.g. for pairs of first companion matrices, the situation is different from the example given above. For these pairs $A_{1}, Z_{1}$, the following holds true: If $m_{2}$ is a non-negative integer such that $\left(A_{1} \oplus O_{m_{2}}, Z_{1} \oplus O_{m_{2}}\right) \in \mathcal{C}\left(m_{1}+m_{2}\right)$, then also $\left(A_{1}, Z_{1}\right) \in \mathcal{C}\left(m_{1}\right)$. We may conclude that for such a pair, extending with zeroes does not produce the property of simultaneous reduction to complementary triangular forms, unless the pair had this property from the beginning.

As was mentioned before, the problem of complementary triangular forms after extensions with zeroes comes up in the infinite-dimensional setting. The following result is obtained there as a by-product.

Proposition 8. If the pair of $m_{1} \times m_{1}$ matrices $A_{1}, Z_{1}$ admits simultaneous reduction to complementary triangular forms after extension with zeroes, then $\left(A_{1} \oplus O_{m_{2}}, Z_{1} \oplus\right.$ $\left.O_{m_{2}}\right) \in \mathcal{C}\left(m_{1}+m_{2}\right)$ for a non-negative integer $m_{2}$ that satisfies the estimate

$$
m_{2} \leq 8 m_{1}^{2}-3 m_{1} .
$$

Even in the general case, the estimate in Proposition 8 is probably not sharp, although improved estimates have not been found yet.

3. Bounded operators. In this section, the notion of complementary triangular forms for pairs of bounded operators on an infinite-dimensional Banach space is considered. A precise description of what is meant by a bounded linear operator in upper triangular form is given in terms of so-called maximal nests of invariant subspaces. A nest of subspaces is a collection of subspaces which is linearly ordered by inclusion. Maximal 
nests of subspaces are nests that are not properly contained in any other nest. Further, a subspace $M$ is invariant for the linear operator $A$ if $x \in M$ implies $A x \in M$, so $A M \subseteq M$.

A linear operator $A$ acting on a Banach space $X$ is called upper triangular with respect to a maximal nest $\mathcal{M}$ of subspaces if $\mathcal{M}$ consists of subspaces that are invariant for $A$. To illustrate the definition, we give two examples. The first example is very simple: Let

$$
U=\left(\begin{array}{ccccc}
0 & 1 & 0 & 0 & \ldots \\
0 & 0 & 1 & 0 & \cdots \\
0 & 0 & 0 & 1 & \cdots \\
0 & 0 & 0 & 0 & \cdots \\
\vdots & \vdots & \vdots & \vdots & \ddots
\end{array}\right)
$$

denote the backward shift on $l_{2}\left(\mathbb{Z}^{+}\right)$. If $\left\{e_{k} \mid k \in \mathbb{Z}^{+}\right\}$denotes the standard basis in $l_{2}\left(\mathbb{Z}^{+}\right)$, then $U$ is upper triangular with respect to the maximal nest of subspaces

$$
\mathcal{M}=\left\{\operatorname{span}\left\{e_{1}, \ldots, e_{k}\right\} \mid k \in \mathbb{Z}^{+}\right\} \cup\left\{(0), l_{2}\left(\mathbb{Z}^{+}\right)\right\} .
$$

The second example, which is quite different from the finite-dimensional setting, is the Volterra integration operator $V$, acting on $L_{2}(0,1)$. It is given by

$$
V f(x)=\int_{0}^{x} f(t) d t,
$$

and it is upper triangular with respect to the continuous maximal nest of subspaces

$$
\mathcal{M}=\left\{L_{2}(\tau, 1) \mid 0 \leq \tau \leq 1\right\} .
$$

Indeed, the subspaces $(0 \leq \tau \leq 1)$

$$
L_{2}(\tau, 1)=\left\{f \mid f \in L_{2}(0,1), f=0 \text { a.e. on }(0, \tau)\right\}
$$

are invariant for $V$. In fact, this is the only maximal invariant nest of subspaces for $V$. hence $V$ is unicellular; see for example [18] or [27].

It is well-known that each compact operator on a Banach space has a maximal nest of invariant subspaces; see [1]. This result can be seen as an infinite-dimensional analogue to Schur's theorem. Recall that an operator is compact if it maps the unit ball into a compact set. Further, a bounded operator $A$ is quasinilpotent if $\sigma(A)=\{0\}$. The analogue to McCoy's theorem for pairs of compact operators on an infinite-dimensional Banach space is provided by the following result from [22].

Theorem 9. Let $A$ and $Z$ be compact operators acting on a Banach space $X$. Then $A$ and $Z$ have a common maximal nest of invariant subspaces if and only if for each polynomial $p(\lambda, \mu)$ in the non-commuting variables $\lambda$ and $\mu$, the compact operator $p(A, Z)(A Z-$ $Z A)$ is quasinilpotent.

Before we define complementary triangular forms for a pair of bounded operators on a Banach space, we return to the finite matrix case: A pair of $m \times m$ matrices $A, Z$ admits simultaneous reduction to complementary triangular forms if and only if there exists a collection of projections $\mathcal{P}=\left\{P_{k} \mid 0 \leq k \leq m\right\}$, such that the collections $\left\{\operatorname{Ran} P_{k} \mid 0 \leq k \leq m\right\}$ and $\left\{\operatorname{Ker} P_{k} \mid 0 \leq k \leq m\right\}$ are maximal nests of invariant subspaces for $A$ and $Z$ respectively. With the natural ordering on projections, the collection $\mathcal{P}$ of projections is a maximal nest of projections. 
In order to extend the notion of complementary triangular forms to pairs of bounded operators on an infinite-dimensional Banach space $X$, we need to consider nests of projections on $X$. In Chapter 4 of [29], it is explained that the most straightforward definition that comes to mind, namely that of maximal nests of projections, does not really work. One needs the somewhat more restricted notion of a simple nest of projections. In its definition below, strong suprema and infima are considered. The strong supremum $P_{0}$ of a nest of projections $\mathcal{P}$ is an upper bound for the nest such that

$$
\begin{gathered}
\operatorname{cl}(\bigcup\{\operatorname{Ran} P \mid P \in \mathcal{P}\})=\operatorname{Ran} P_{0}, \\
\bigcap\{\operatorname{Ker} P \mid P \in \mathcal{P}\}=\operatorname{Ker} P_{0} .
\end{gathered}
$$

If the strong supremum for $\mathcal{P}$ exists, it is denoted by $\bigvee \mathcal{P}$. The definition of the strong infimum of the nest $\mathcal{P}$ is similar. If it exists, it is denoted by $\wedge \mathcal{P}$. A nest of projections $\mathcal{P}$ is called simple if the following properties are satisfied.

1. The trivial projections $O$ and $I$ are in $\mathcal{P}$.

2. For any non-empty subnest $\mathcal{P}_{1} \subseteq \mathcal{P}$, the strong supremum $\bigvee \mathcal{P}_{1}$ and the strong infimum $\bigwedge \mathcal{P}_{1}$ exist and are in $\mathcal{P}$.

3. If $P_{1}, P_{2} \in \mathcal{P}, P_{1}<P_{2}$, and there does not exist $P \in \mathcal{P}$ with $P_{1}<P<P_{2}$, then $\operatorname{rank}\left(P_{2}-P_{1}\right)=1$.

With this notion available to us, we introduce complementary triangular forms for pairs of bounded operators on a Banach space. It is convenient to give such a definition by describing the relevant collection of pairs of bounded operators.

The collection $\mathcal{C}(X)$ consists of pairs of bounded operators $A, Z$ acting on $X$ with the following property: There exists a simple nest of projections $\mathcal{P}$ on $X$, such that $A P=P A P$, and $P Z=P Z P$ for all $P \in \mathcal{P}$.

Theorem 4.1.3 of [29] states that a nest of projections is simple if and only if the collections $\{\operatorname{Ran} P \mid P \in \mathcal{P}\}$ and $\{\operatorname{Ker} P \mid P \in \mathcal{P}\}$ are maximal nests of subspaces. It follows that if $\mathcal{P}$ is a simple nest of projections such that $A P=P A P$ and $P Z=P Z P$ for all $P \in \mathcal{P}$, then $\{\operatorname{Ran} P \mid P \in \mathcal{P}\}$ and $\{\operatorname{Ker} P \mid P \in \mathcal{P}\}$ are maximal nests of invariant subspaces for $A$ and $Z$ respectively.

There are many well-known examples of simple nests of subspaces. First of all, each maximal nest of orthoprojectors on a Hilbert space is simple. In particular, each orthonormal basis $\left\{e_{n}\right\}_{n=1}^{\infty}$ in a Hilbert space induces a simple nest of orthoprojections $\left\{P_{n}\right\}_{n=0}^{\infty} \cup\{I\}$, where the non-trivial orthoprojections are given by

$$
P_{n} x=\sum_{k=1}^{n}\left(x, e_{k}\right) e_{k}, \quad n \in \mathbb{Z}^{+} .
$$

Further, the nest of projections induced by a Schauder basis in a Banach space forms a simple nest of projections.

If $A$ and $Z$ are finite rank operators acting on $X$, then there exists a finite rank projection $P$ such that $A=P A P$ and $Z=P Z P$. Moreover, the restrictions $A_{1}, Z_{1}$ of $A$, $Z$, respectively, to the finite-dimensional subspace $\operatorname{Ran} P$ can be identified with a pair of finite matrices of order $\operatorname{rank} P$. 
If a pair of finite rank operators assumes complementary triangular forms with respect to a simple nest, the question remains whether anything can be said about the pair of restrictions $A_{1}, Z_{1}$. It turns out that the notion of simultaneous reduction to complementary triangular forms after extension with zeroes plays a role here. This is shown by Proposition 10 below (see Chapter 5 in [29]).

The proposition involves the following geometric property for a Banach space $X$. A Banach space $X$ has the Simple Nest Property if for each subspace $Y \subseteq X$ of finite codimension, there exists a bounded simple nest of projections acting on $Y$. Note that Hilbert spaces and Banach spaces with a Schauder basis have the Simple Nest Property. It is not known to the author whether there exist Banach spaces without the Simple Nest Property. Taking into account [14], this problem seems to be rather interesting.

Proposition 10. Let $A$ and $Z$ be finite rank operators acting on an infinite-dimensional Banach space $X$. Let $A_{1}$ and $Z_{1}$ be the restrictions to $\operatorname{Ran} P$ as above. If $(A, Z) \in$ $\mathcal{C}(X)$, then the pair $A_{1}, Z_{1}$ admits simultaneous reduction to complementary triangular forms after extension with zeroes. Moreover, if the Banach space $X$ has the Simple Nest Property, then the converse also holds.

Acknowledgements. The author would like to thank H. Bart for his helpful comments and suggestions.

\section{References}

[1] N. Aronszajn and K. T. Smith, Invariant subspaces of completely continuous operators, Ann. of Math. 60 (1954), 345-350.

[2] H. Bart, Transfer functions and operator theory, Linear Algebra Appl. 84 (1986), 33-61.

[3] H. Bart, I. Gohberg and M. A. Kaashoek, Minimal Factorization of Matrix and Operator Functions, Oper. Theory: Adv. Appl. I, Birkhäuser, Basel, 1979.

44 H. Bart and H. Hoogland, Complementary triangular forms of pairs of matrices, realizations with prescribed main matrices, and complete factorization of rational matrix functions, Linear Algebra Appl. 103 (1988), 193-228.

[5] H. Bart and L. G. Kroon, Companion based matrix functions: description and minimal factorization, Linear Algebra Appl. 248 (1996), 1-46.

[6] --, - Factorization and job scheduling: a connection via companion based rational matrix functions, ibid., to appear.

[7] - - - Variants of the two machine flow shop problem, European J. Oper. Res., to appear.

[8] H. Bart and G. Ph. A. Thijsse, Complementary triangular forms of upper triangular Toeplitz matrices, in: Oper. Theory: Adv. Appl. 40, Birkhäuser, 1989, 133-149.

[9] - - -, Complementary triangular forms of nonderogatory, Jordan and rank one matrices, Report 9003/B, Econometric Institute, Erasmus University Rotterdam, 1990.

[10] - - - Eigenspace and Jordan-chain techniques for the description of complementary triangular forms, Report 9353/B, Econometric Institute, Erasmus University Rotterdam, 1993.

[11] H. Bart and H. K. Wimmer, Simultaneous reduction to triangular and companion forms of pairs of matrices: the case $\operatorname{rank}(I-A Z)=1$, Linear Algebra Appl. 150 (1991), 443-461.

[12] H. Bart and R. A. Zuidwijk, Triangular forms after extensions with zeroes, submitted. 
[13] M. P. Drazin, J. W. Dungey and K. W. Gruenberg, Some theorems on commutative matrices, J. London Math. Soc. 26 (1951), 221-228.

[14] P. Enflo, A counterexample to the approximation property in Banach spaces, Acta Math. 130 (1973), 309-317.

[15] S. Friedland, Pairs of matrices which do not admit a complementary triangular form, Linear Algebra Appl. 150 (1990), 119-123.

[16] G. Frobenius, Über vertauschbare Matrizen, Sitz.-Ber. Akad. Wiss. Berlin 26 (1896), 601-614.

[17] F. J. Gaines and R. C. Thompson, Sets of nearly triangular matrices, Duke Math. J. 35 (1968), 441-453.

[18] I. Gohberg and M. G. Krein, Theory and Applications of Volterra Operators in Hilbert Space, Transl. Math. Monographs 24, A.M.S, Providence, R.I., 1969.

[19] T. J. Laffey, Simultaneous triangularization of a pair of matrices, J. Algebra 44 (1977), 550-557.

[20] - Simultaneous triangularization of matrices-low rank cases and the nonderogatory case, Linear and Multilinear Algebra 6 (1978), 269-305.

[21] - Simultaneous reduction of sets of matrices under similarity, Linear Algebra Appl. 84 (1986), 123-138.

[22] C. Laurie, E. Nordgren, H. Radjavi and P. Rosenthal, On triangularization of algebras of operators, J. Reine Angew. Math. 327 (1981), 143-155.

[23] P. Lancaster and M. Tismenetsky, The Theory of Matrices, Second Edition with Applications, Academic Press, Orlando, Fla., 1985.

[24] N. H. McCoy, On the characteristic roots of matric polynomials, Bull. Amer. Math. Soc. 42 (1936), 592-600.

[25] G. J. Murphy, Triangularizable algebras of compact operators, Proc. Amer. Math. Soc. 84 (1982), 354-356.

[26] H. Radjavi, A trace condition equivalent to simultaneous triangularizability, Canad. J. Math. 38 (1986), 376-386.

[27] J. R. Ringrose, Non-Self-Adjoint Compact Linear Operators, van Nostrand, New York, 1971.

[28] S. H. Tan and J. Vandewalle, On factorizations of rational matrices, IEEE Trans. Circuits and Systems 35 (1988), 1179-1181.

[29] R. A. Zuidwijk, Complementary triangular forms for pairs of matrices and operators, doctoral thesis, 1994.

[30] --, Quasicomplete factorizations for rational matrix functions, Integral Equations Operator Theory, to appear.

[31] R. A. Zuidwijk, H. Bart and L. Kroon, Quasicomplete factorization and the two machine flow shop problem, Report 9632/B, Econometric Institute, Erasmus University Rotterdam, 1996.

Editorial note: See also Ph. Thijsse, Spectral criteria for complementary triangular forms, Integral Equations Operator Theory 27 (1997), 228-251. 